\title{
MORE FIBONACCI VARIETIES
}

\author{
Ann Chi Kim, B.H. Neumann, and A.H, Rhemtulla
}

\begin{abstract}
The Fibonacci varieties introduced by Ann Chi Kim, Buzz. Austral. Math. Soc. 19 (1978), 191-196 (1979), are here generalised in an obvious way suggested by the work of D.L. Johnson, J.W. Wamsley, and D. Wright, Proc. London Math. Soc. (3) 29 (1974), 577-592. The underlying groups of algebras in these varieties are studied, and are shown to be abelian when the algebras are generated by a single element, and in general are found to be extensions of central subgroups by groups of finite exponent.
\end{abstract}

\section{Introduction}

We consider algebraic systems, or briefly algebras, that are groups with an aditional unary operation. We use conventional group notation: $x \cdot y, x^{-1}, e$ for the product, inverse, and unit element, respectively; the additional unary operation is denoted by $\phi$ and written as a right-hand operator. In addition to the group laws, which we assume but do not here specify, we assume the laws

$$
(x \cdot y) \phi=x \phi \cdot y \phi, \quad x^{-1} \phi=(x \phi)^{-1}, \quad e \phi=e
$$

(of which the first is well known to imply the other two);

Received I May 1980. The second author is indebted, and deeply grateful, to Professor Narain D. Gupta and the Department of Mathematics and Astronomy of the University of Manitoba, Winnipeg, for hospitality during the autumn semester 1979, during which this work was carried out. He also thanks the many colleagues and friends who, by discussion in and after talks he gave in many places, have contributed many useful ideas; especially Narain D. Gupta, Ileter M. Neumann, R. Padmanabhan, R. Quackenbush, Sheila Oates Williams, Barry Wolk. 


$$
x \phi^{m}=x \phi^{m-1} \cdot x \phi^{m-2} \cdot \ldots \cdot x \phi \cdot x
$$

and

$$
x \phi^{n}=x
$$

Here $m$ and $n$ are integers, with $m \geq 2$ and $n \geq 0$; the case $n=0$ means that the law (1.3) is trivially satisfied and can be omitted.

The inspiration for this set-up is in the Fibonacci groups studied, inter alia, by Johnson, Wamsley, and Wright [3] (see also Chalk and Johnson [1] for further references). These authors define the Fibonacci groups

$$
\begin{array}{r}
F(m, n)=\operatorname{gp}\left(a_{1}, a_{2}, \ldots, a_{n} ; a_{i+m}=a_{i+m-1} \cdot a_{i+m-2} \cdot \ldots \cdot a_{i},\right. \\
\quad i=1,2, \ldots, n, \text { suffixes taken modulo } n),
\end{array}
$$

together with the abelianised groups

$$
\begin{aligned}
A(m, n)=F(m, n) / F(m, n)^{\prime} & =\operatorname{gp}\left(a_{1}, a_{2}, \ldots, a_{n} ;\right. \\
a_{i+m} & =a_{i+m-1} \cdot a_{i+m-2} \cdot \ldots \cdot a_{i}, a_{i} \cdot a_{j}=a_{j} \cdot a_{i}, \\
i, j & =1,2, \ldots, n, \text { suffixes taken modulo } n) .
\end{aligned}
$$

In order to allow also for the case $n=0$, which makes not much sense in the Johnson et al. set-up, but is of interest in ours, we also define

$$
\begin{aligned}
F(m, 0)= & \operatorname{gp}\left(\ldots, a_{-1}, a_{0}, a_{1}, a_{2}, \ldots ; a_{i+m}=a_{i+m-1} \cdot a_{i+m-2} \cdot \ldots \cdot a_{i},\right. \\
A(m, 0)= & F(m, 0) / F(m, 0)^{\prime}=\operatorname{gp}\left(\ldots, a_{-1}, a_{0}, a_{1}, a_{2}, \ldots ;\right. \\
& \left.a_{i+m}=a_{i+m-1} \cdot a_{i+m-2} \cdot \ldots \cdot a_{i}, a_{i} \cdot a_{j}=a_{j} \cdot a_{i}, \text { for all } i, j\right) .
\end{aligned}
$$

It is not difficult to see that $F(m, 0)$ is a free group of rank $m$, and $A(m, 0)$ a free abelian group of rank $m$, both freely generated by $a_{0}, a_{1}, \ldots, a_{m-1}$, or by any other set of $m$ consecutive $a_{i}$.

These groups $F(m, n)$ and $A(m, n)$ have an obvious automorphism $\alpha$, say, of order $n$, defined by

$$
a_{i} \alpha=a_{i+1} \text { (suffixes modulo } n \text { ). }
$$

Our operator $\phi$ imitates the action of $\alpha$, but not only on a preferred set of generators of the group involved, but on all its elements. This imposes severe restrictions on the groups underlying our algebras; on the 
other hand, our approach leads to some new phenomena: the Fibonacci groups are clearly generated by $a_{1}$ and its images under powers of $\alpha$, and thus correspond to monogenic (that is one-generator) algebras in our context. We shall, however, also look at algebras with more than one generator.

We denote the variety of algebras defined by the laws (1.1)-(1.3) by $\underline{\underline{\mathrm{V}}}(m, n)$. In [4], one of us studied the varieties $\underline{\underline{\mathrm{V}}}(2, n)$ [there denoted simply by $\left.\stackrel{\mathrm{V}}{G}_{n}\right]$, and determined the free monogenic algebras of these varieties, for all $n \geq 1$. In the present paper we deal with the cases $m \geq 3$, and we specifically do not exclude the case $n=0$, because this involves the weakest assumptions and thus greatest generality. We also pay special attention to the free algebras of $\underline{\underline{V}}(m, n)$ and to their underlying groups. Denote the free algebra on $d$ generators in $\underline{\underline{V}}(m, n)$ by $A_{d}(m, n)$ and its underlying group by $G_{d}(m, n)$. Our results then are as follows :

THEOREM 1. The group $G_{1}(m, n)$ underlying the monogenic free algebra of $\underline{\underline{\mathrm{V}}}(m, n)$ is abelian, and isomorphic to $A(m, n)$ (in the notation of Johnson, Wamsley, and Wright [3] - see above).

A proof that $A(m, n)$ is finite when $m \geq 2, n \geq 1$, and formulae for the orders of these groups, are given by Johnson [2]. It has already been remarked that $A(m, 0)$ is free abelian of rank $m$, hence infinite.

THEOREM 2. The groups $G_{d}(3, n)$ underlying the d-generator free algebras of $\underline{v}(3, n)$ are abelian, for all $d \geq 1$ and all $n \geq 0$; in fact the groups undertying arbitrary algebras in $\mathrm{V}(3, n)$ are abelian.

This carries commutativity of the underlying groups from $m=2$ (see the Lemma in [4]) over to $m=3$; but there the commutativity stops:

THEOREM 3. The groups $G$ underlying algebras $A$ in $\underline{\underline{V}}(m, n)$ satisfy "centre-by exponent $m-1$ " low

$$
\left[x^{m-1}, y\right]=e,
$$

where we use the customary commutator notation $[x, y]=x^{-1} \cdot y^{-1} \cdot x \cdot y$. Thus such a group $G$ has a subgroup $Z$ in its centre such that $G / Z$ lies in the Burnside variety $\stackrel{B}{m}-1_{m}$ of groups of exponent $m-1$. Conversely 


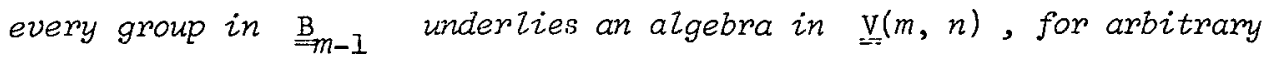
$n \geq 0$. Thus when $m \geq 4$, there are non-abelian groups that underly algebras in $\underline{\underline{v}}(m, n)$.

Note, however, that the $l$ aw $(1.4)$ is not sufficient to characterise the groups underlying algebras in $\underline{\underline{V}}(m, n)$, especially when $n \geq 1$.

\section{Preliminary results}

It is clear that if the integer $p$ divides the integer $q$, then the law $x \phi^{p}=x$ implies the law $x \phi^{q}=x$. Hence we have the following fact:

LEMMA 1. For all integers $p, q$ such that $p$ divides $q$, and all $m$,

$$
\underline{\underline{V}}(m, 1) \subseteq \underline{\underline{V}}(m, p) \subseteq \underline{\underline{V}}(m, q) \subseteq \underline{\underline{V}}(m, 0)
$$

Next we show that $\phi$ is not only an endomorphism, but an automorphism of the underlying group of every algebra in our varieties.

LEMMA 2. Let $\mathrm{A}$ be an arbitrary algebra in $\underline{\underline{\mathrm{V}}}(m, n)$ and $G$ its underlying group; then $\phi$ acts as an automorphism of $G$.

Proof. The morphism property of $\phi$ is guaranteed by the laws (1.1), so we need only show that $\phi$ is one-to-one and onto. If $n>0$, this is obvious, as then $\phi^{n}$ is the identity mapping of $G$, the carrier (or set of elements) of $G$, which is also, of course, the carrier of A. For arbitrary $n \geq 0$ we use the law (1.2), to write

$$
\begin{aligned}
x & =(x \phi)^{-1} \cdot\left(x \phi^{2}\right)^{-1} \cdot \ldots \cdot\left(x \phi^{m-1}\right)^{-1} \cdot x \phi^{m} \\
& =\left(x^{-1} \cdot x^{-1} \phi \cdot \ldots \cdot x^{-1} \phi^{m-2} \cdot x \phi^{m-1}\right) \phi .
\end{aligned}
$$

This shows that every element $x$ is an image under $\phi$; thus $\phi: G \rightarrow G$ is onto $G$. Also, if $x \phi=y \phi$, then also $x \phi^{2}=y \phi^{2}$, and so on; thus

$$
\begin{aligned}
x= & (x \phi)^{-1} \cdot\left(x \phi^{2}\right)^{-1} \cdot \ldots \cdot\left(x \phi^{m-1}\right)^{-1} \cdot x \phi^{m} \\
& (y \phi)^{-1} \cdot\left(y \phi^{2}\right)^{-1} \cdot \ldots \cdot\left(y \phi^{m-1}\right)^{-1} \cdot y \phi^{m}=y .
\end{aligned}
$$

This shows that $\phi$ is also one-to-one. // 


\section{Proof of Theorem 1}

From now on we use the morphism property (1.1) of $\phi$, and of powers of $\phi$, without further mention. Apply $\phi$ to both sides of (1.2) to get

$$
x \phi^{m+1}=x \phi^{m} \cdot x \phi^{m-1} \cdot \ldots \cdot x \phi .
$$

Then

$$
\begin{aligned}
x \phi^{m+1} \cdot x & =x \phi^{m} \cdot x \phi^{m-1} \cdot \ldots \cdot x \phi \cdot x \\
& =x \phi^{m} \cdot x \phi^{m} .
\end{aligned}
$$

Now we evaluate $(x \phi \cdot y) \phi^{m}$ by using (1.2) again, with $x \phi \cdot y$ and then $y \cdot x$ in place of $x$ :

$$
\begin{aligned}
(x \phi \cdot y) \phi^{m} & =(x \phi \cdot y) \phi^{m-1} \cdot(x \phi \cdot y) \phi^{m-2} \cdot \ldots \cdot x \phi \cdot y \\
& =x \phi^{m} \cdot y \phi^{m-1} \cdot x \phi^{m-1} \cdot y \phi^{m-2} \cdot \ldots \cdot x \phi \cdot y \\
& =x \phi^{m} \cdot(y \cdot x) \phi^{m-1} \cdot(y \cdot x) \phi^{m-2} \cdot \ldots \cdot(y \cdot x) \cdot x^{-1} \\
& =x \phi^{m} \cdot(y \cdot x) \phi^{m} \cdot x^{-1} \\
& =x \phi^{m} \cdot y \phi^{m} \cdot x \phi^{m} \cdot x^{-1} . \\
& x \phi^{m+1} \cdot y \phi^{m}=x \phi^{m} \cdot y \phi^{m} \cdot x \phi^{m} \cdot x^{-1} .
\end{aligned}
$$

Put $y=x \phi$ to get

$$
x \phi^{m+1} \cdot x \phi^{m+1} \cdot x=x \phi^{m} \cdot x \phi^{m+1} \cdot x \phi^{m}
$$

and apply $(3.2)$ :

$$
x \phi^{m+1} \cdot x \phi^{m} \cdot x \phi^{m}=x \phi^{m} \cdot x \phi^{m+1} \cdot x \phi^{m}
$$

Tris then gives

$$
\left(3.4_{1}\right) \quad x \phi^{m+1} \cdot x \phi^{m}=x \phi^{m} \cdot x \phi^{m+1} .
$$

We now proceed by induction. Assume that we have, for some $j \geq 1$, the laws
$\left(3 .{ }^{4}\right)$
$x \phi^{m+j} \cdot x \phi^{m}=x \phi^{m} \cdot x \phi^{m+j}$
$\left(3.5_{j}\right)$
$x \phi^{m+j} \cdot x \phi^{m+1}=x \phi^{m+1} \cdot x \phi^{m+j}$ 
We note that $\left(3.4_{1}\right)$ has just been proved, and that $\left(3.5_{1}\right)$ is trivially satisfied. Applying $\phi$ to $\left(3.4_{j}\right)$ immediately gives $\left(3.5_{j+1}\right)$. To prove also $\left(3.4_{j+1}\right)$, we apply $\left(3.4_{j}\right)$ to $x \phi \cdot x$ in place of $x$ :

$$
(x \phi \cdot x) \phi^{m+j} \cdot(x \phi \cdot x) \phi^{m}=(x \phi \cdot x) \phi^{m} \cdot(x \phi \cdot x) \phi^{m+j} .
$$

Thus

$$
x \phi^{m+j+1} \cdot x \phi^{m+j} \cdot x \phi^{m+1} \cdot x \phi^{m}=x \phi^{m+1} \cdot x \phi^{m} \cdot x \phi^{m+j+1} \cdot x \phi^{m+j} .
$$

Applying $\left(3.5_{j}\right)$ and $\left(3.5_{j+1}\right)$, we shift $x \phi^{m+1}$ on the left-hand side to the left, then cancel it:

$$
x \phi^{m+j+1} \cdot x \phi^{m+j} \cdot x \phi^{m}=x \phi^{m} \cdot x \phi^{m+j+1} \cdot x \phi^{m+j} .
$$

Here we use $\left(3.4_{j}\right)$ to shift $x \phi^{m+j}$ to the right on the left-hand side, then cancel it, giving finally

$$
\left(3 \cdot 4_{j+1}\right) \quad x \phi^{m+j+1} \cdot x \phi^{m}=x \phi^{m} \cdot x \phi^{m+j+1} \text {. }
$$

By induction $\left(3.4_{j}\right)$ is then established for all positive $j$. Now given integers $k, l$ with $k>l$, we apply $\phi^{z-m}$ to $\left(3.4_{k-l}\right)$ to get

$$
x \phi^{k} \cdot x \phi^{2}=x \phi^{2} \cdot x \phi^{k}
$$

As this is symmetric in $k, l$ and the case $k=l$ is trivial, we have proved the first statement in the following lemma:

LEMMA 3. In every variety $\underline{\underline{\mathrm{V}}}(m, n)$ the low $(3.6)$ is valid for arbitrary integers $k, Z$. If $A$ is a monogenic algebra in $\underline{V}(m, n)$, then its underlying group $G$ is abelian.

The second statement follows from the fact that if $A$ is generated, qua algebra, by the single element $a$, then its underlying group $G$ is generated, qua group, by the set $\left\{a \phi^{j} \mid j\right.$ an integer $\}$, and even, because of the law (1.2), by the set $\left\{a \phi^{j} \mid 0 \leq j \leq m-1\right\}$. //

Now the proof of Theorem 1 is the same, mutatis mutandis, as that of the Theorem in [4], and we omit it. 


\section{Proof of Theorem 2}

We now turn to the special case $m=3$, and prove commutativity of the underlying groups in this case. The law (1.2) becomes

$$
\text { (4.1) } \quad x \phi^{3}=x \phi^{2} \cdot x \phi \cdot x ;
$$

and we shall proceed without the law (1.3), that is to say, we work in the variety $\underline{\underline{v}}(3,0)$, of which all $\underline{\underline{V}}(3, n)$ are, as we know, subvarieties.

We begin by applying (4.1) to a product $x \cdot z$ :

$$
(x \cdot z) \phi^{3}=(x \cdot z) \phi^{2} \cdot(x \cdot z) \phi \cdot x \cdot z,
$$

or

$$
x \phi^{3} \cdot z \phi^{3}=x \phi^{2} \cdot z \phi^{2} \cdot x \phi \cdot z \phi \cdot x \cdot z
$$

Applying (4.1) to $x$ and $z$ separately, and multiplying, we also get

$$
x \phi^{3} \cdot z \phi^{3}=x \phi^{2} \cdot x \phi \cdot x \cdot z \phi^{2} \cdot z \phi \cdot z
$$

Comparison of (4.21) and (4.22) then gives, after cancelling $x \phi^{2}$ and $z$,

$$
z \phi^{2} \cdot x \phi \cdot z \phi \cdot x=x \phi \cdot x \cdot z \phi^{2} \cdot z \phi
$$

Here we put $z \phi=y$, and note that, as a consequence of Lemma $2, y$ ranges with $z$ over the whole carrier of our algebra; thus we get the law

$$
y \phi \cdot x \phi \cdot y \cdot x=x \phi \cdot x \cdot y \phi \cdot y,
$$

or

$$
(y \cdot x) \phi \cdot y \cdot x=x \phi \cdot x \cdot y \phi \cdot y
$$

Now we define a new operation $\psi$ by

$$
x \psi=x \phi \cdot x .
$$

Then (4.3) says that

$$
(y \cdot x) \psi=x \psi \cdot y \psi
$$

that is to say, $\psi$ is an antimorphism: it reverses the order of the factors in a product. Then $\psi^{2}$ must restore that order, and be a morphism. Now $x \psi^{2}$ is, in terms of $\phi$, 


$$
\begin{aligned}
x \psi^{2} & =(x \phi \cdot x) \phi \cdot x \phi \cdot x \\
& =x \phi^{2} \cdot x \phi \cdot x \phi \cdot x \\
& =x \phi^{2} \cdot x \phi \cdot x \cdot x \phi,
\end{aligned}
$$

where we have used the law (3.6). Applying (4.1) we get

$$
x \psi^{2}=x \phi^{3} \cdot x \phi \text {. }
$$

Now as $\psi^{2}$ is a morphism, so is $\psi^{2} \phi^{-1}$, which, using (4.4), is given by

$$
x \psi^{2} \phi^{-1}=x \phi^{2} \cdot x \text {. }
$$

Hence, applying this to the product $x \cdot z$, we have

$$
(x \cdot z) \phi^{2} \cdot x \cdot z=x \phi^{2} \cdot x \cdot z \phi^{2} \cdot z
$$

or

$$
x \phi^{2} \cdot z \phi^{2} \cdot x \cdot z=x \phi^{2} \cdot x \cdot z \phi^{2} \cdot z
$$

Cancelling $x \phi^{2}$ on the left, $z$ on the right, and putting $z \phi^{2}=y$ (which again ranges with $z$ over the whole carrier of our algebra), we finally have the commutative law

$$
y \cdot x=x \cdot y,
$$

and Theorem 2 follows.

$$
/ /
$$

\section{Proof of Theorem 3}

We return to the case of general $m$, and again dispense with the law (1.3), that is to say, we work in $\underline{\underline{v}}(m, 0)$. We deduce from (3.2) the law

$$
\left(x^{-1} \cdot x \phi\right) \phi^{m}=\left(x \phi^{m}\right)^{-1} \cdot x \phi^{m+1}=x \phi^{m} \cdot x^{-1} \text {. }
$$

We digress by noting that this implies that for any element, say $g$, of the carrier of an algebra in $\mathrm{V}(m, n)$,

$$
g \phi^{m}=g
$$

is equivalent with

$$
g \phi=g:
$$

LEMMA 4. An element $g$ of the carrier of an algebra in $\underline{\underline{V}}(m, n)$ is 
fixed by $\phi^{m}$ if, and only if, $g$ is fixed by $\phi$.

COROLLARY. The varieties $\underline{\underline{\mathrm{V}}}(m, m)$ and $\underline{\underline{\mathrm{V}}}(m, 1)$ coincide; and they coincide with all varieties $\underline{\mathrm{V}}\left(m, m^{\prime}\right)$, where $m^{\prime}$ divides $m$.

The last statement follows from Lemma 1 . Now we re-write (3.3) in the form

$$
\left(x \phi^{m}\right)^{-1} \cdot x \phi^{m+1} \cdot y \phi^{m}=y \phi^{m} \cdot x \phi^{m} \cdot x^{-1}
$$

Using (5.1), this gives

$$
\left(x^{-1} \cdot x \phi\right) \phi^{m} \cdot y \phi^{m}=y \phi^{m} \cdot\left(x^{-1} \cdot x \phi\right) \phi^{m},
$$

and applying $\phi^{-m}$, we finally get

$$
x^{-1} \cdot x \phi \cdot y=y \cdot x^{-1} \cdot x \phi
$$

This law says that $x^{-1} \cdot x \phi$ commutes with all elements, in other words: is central in the underlying group $G$ of the algebra $A$ under consideration.

The set $\left\{g^{-1} \cdot g \phi \mid g \in G\right\}$, where $G$ is the carrier of $G$ (and of A ), generates a subgroup $Z$ of $G$, and we have proved, by (5.2):

LEMMA 5. The subgroup $Z$ of $G$ lies in the centre of $G$.

It is not difficult to verify that the carrier $Z$ of $Z$ is, in fact, the set $\left\{g^{-1} \cdot g \phi \mid g \in G\right\}$ we have used to generate $Z$; and that $Z$ is the underlying group of a subalgebra $T$ of $A$. In fact $T$ is the kernel of the natural epimorphism of $A$ onto an algebra $A_{1}$, with underlying group $G_{1}=G / Z$, where $\phi$ acts on $G_{1}$ as the identity automorphism. The algebra $A_{1}$ is obtained from $A$ by introducing the law

$$
x \phi=x,
$$

or strengthening the law (1.3) to (5.3). With this law, (1.2) becomes

$$
x=x^{m},
$$

or

$$
x^{m-1}=e
$$

the "Burnside" law with exponent $m-1$. Thus $G_{1}$ lies in the Burnside 
variety $\stackrel{B}{=}_{m-1}$ of groups of exponent $m-I$. Conversely, if $G_{1}$ is an arbitrary group of exponent $m-1$, and if we define the operator $\phi$ as the identity automorphism of $G_{1}$, then all the laws $(1.1),(1.2),(1.3)$ are trivially satisfied, so that we get an algebra $A_{1}$ with $G_{1}$ as its underlying group, and $A_{1}$ belongs to $\underline{v}(m, 1)$ (and thus to all $\underline{\underline{V}}(m, n)$, with $n \geq 0$ ). Theorem 3 follows. $/ /$

If we take $G_{1}$ in particular as the free $d$-generator group $B_{d, m-I}$ of $\mathrm{B}_{m-1}$, we thus clearly obtain the group $G_{d}(m, 1)$ underlying the free $d$-generator algebra $A_{d}(m, 1)$ of $\underline{\underline{\mathrm{v}}}(m, 1)$. As the variety $\underline{\underline{\mathrm{v}}}(m, 1)$ is obtained from $\underline{\underline{V}}(m, n)$ by strengthening the law (1.3) to (5.3), the free d-generator algebra $A_{d}(m, n)$ of $\underline{V}(m, n)$ maps epimorphically on $A_{d}(m, 1)$, and thus its underlying group $G_{d}(m, n)$ maps epimorphically on this Burnside group $B_{d, m-1}=G_{d}(m, 1)$. The kernel is, as we know, a central subgroup $Z_{d}(m, n)$ of $G_{d}(m, n)$, generated by the set $\left\{g^{-1} \cdot g \phi \mid g \in G_{d}(m, n)\right\}$. This depends on the values of the parameters $m, n, d$. For example, we know that for $n=1$, or indeed for any $n$ dividing $m$, this subgroup is trivial (see the Corollary to Lemma 4). We state without proof that at the other extreme, namely $n=0$, this kernel $z_{d}(m, 0)$ is free abelian of rank $d \cdot m$, and that for $n>0$ and finite $d$ it is a finite abelian group.

\section{References}

[1] C.P. Chalk and D.L. Johnson, "The Fibonacci groups. II", Proc. Roy. Soc. Edinburgh Sect. A 77 (1977), 79-86.

[2] D.L. Johnson, "A note on the Fibonacci groups", Israel J. Math. 17 $(1974), 277-282$.

[3] D.L. Johnson, J.W. Wamsley, and D. Wright, "The Fibonacei groups", Proc. London Math. Soc. (3) 19 (1974), 577-594. 
[4] Ann Chi Kim, "Fibonacci varieties", Bull. Austral. Math. Soc. 19 (1978), 191-196 (1979).

Department of Mathematics, Busan National University, Busan,

Korea, 607;

Department of Mathematics, Institute of Advanced Studies, Australian National University, PO Box 4, Canberra, ACT, Australia 2600, and

Division of Mathematics and Statistics, Commonwealth Scientific and Industrial Research Organization, PO Box 1965, Canberra City, ACT, Australia 2601;

Department of Mathematics, University of Alberta, Edmonton,

Alberta,

Canada T6G 2GI. 